\title{
Palliative Care for Pancreatic and Periampullary Cancer
}

\author{
Jennifer A. Perone, M.D. ${ }^{1}$ \\ Taylor S. Riall, M.D., Ph.D ${ }^{2}$ \\ Kelly Olino, M.D. ${ }^{1}$
}

${ }^{1}$ Department of Surgery, The University of Texas Medical Branch, Galveston, Texas and ${ }^{2}$ University of Arizona, Banner-University Medical Center, Tucson, Arizona

Funding: Supported by grants from the UTMB Clinical and Translational Science Award \#UL1TR000071, NIH T-32 Grant \# 5T32DK007639, AHRQ Grant \#1R24HS022134, and Cancer Prevention and Research Institute Grant \#RP140020

\section{Corresponding Author:}

Taylor S. Riall, M.D., Ph.D.

Professor

University of Arizona

Banner-University Medical Center

1501 N Campbell Dr, Rm 4327b

PO Box 245131

Tucson, AZ 85274-5131

Phone: 520-626-6664

Fax: 520-626-7785

Email: tsriall@surgery.arizona.edu 


\section{Synopsis}

Most patients with pancreatic cancer will present with metastatic or locally advanced disease[1-3]. Unfortunately, the majority of patients with localized disease will recur even after multi-modality therapy including surgical resection combined with neoadjuvant or adjuvant chemotherapy. As such, pancreatic cancer patients arrive at a common endpoint where decisions pertaining to palliative care come to the forefront. This chapter summarizes surgical, endoscopic and other palliative techniques for relief of obstructive jaundice, relief of duodenal or gastric outlet obstruction, and relief of pain due to invasion of the celiac plexus. It also introduces the utility of the palliative care triangle in clarifying a patient's and family's goals to guide decision-making and choose "the right treatment, for the right patient, in the right setting." 
Pancreatic cancer remains a highly lethal disease, with nearly $80 \%$ of patients presenting with metastatic or locally advanced disease.[1-3] Modern first-line chemotherapy for metastatic disease (FOLFIRINOX: 5-fluorouracil, leucovorin, irinotecan, and oxaliplatin), improves median survival from 2-4 months to approximately 11 months in phase III trials,[4-7] while patients with unresectable locally advanced disease who receive treatment with chemo-radiation have median survival ranging from 11-15 months.[4, 8-11] The highest median survival rates are 22-26 months in patients who are candidates to undergo surgical resection, with the addition of neoadjuvant or adjuvant therapy further improving survival.[12-15] More recent studies evaluating the impact of neoadjuvant therapy on highly selected patients with resectable disease have yielded median survival times as long as 44 months in patients with R0, node negative disease.[16-18]

Despite these moderate improvements in survival, the overall prognosis remains poor and treatment is associated with the inherent risks of surgery, radiation, and chemotherapy. Given this, multidisciplinary management and shared decision-making are needed at every stage of disease in order to evaluate the prognosis, the performance status of the patient, the availability and success of both surgical and nonsurgical management options, along with an individual patient's preferences.

The pendulum has shifted away from operative palliation in the last two decades. However, as both systemic therapy for metastatic pancreatic cancer and non-surgical palliative options continue to evolve, the effectiveness of palliative therapy options with respect to both the quantity and quality of life must be continually re-evaluateed as new therapeutic options are made available.

In this chapter, we will first focus on the modern concept of palliative care and then discuss the options and outcomes for palliation in pancreatic cancer, focusing on three common problems: 1) relief of obstructive jaundice, 2) relief of duodenal or gastric outlet obstruction, and 3) relief of pain due to invasion of the celiac plexus. Finally, we will discuss issues related to nutrition and managing the sequelae of venous thromboembolic disease. 


\section{Goals and Outcomes of Palliative Care}

Palliative care is described by the World Health Organization (WHO) as: "The total active care of patients whose disease is not responsive to curative treatment. Control of pain, other symptoms, and psychological, social, and spiritual problems, is paramount. The goal of palliative care is achievement of the best quality of life for patients and their families." [25]

Palliative care aims to provide treatment that extends beyond the traditional goals of addressing physical symptoms and survival by integrating the psychosocial and spiritual aspects of patient care. Far from hastening death, early intervention with palliative care results in improved quality of life and at times prolonged survival compared to standard treatment alone.[26] As such, palliative care should be viewed more broadly as part of the spectrum of survivorship and supportive care (Figure 1). Therefore, to be most effective, the concept of palliative care should be introduced early in the course of an illness, in conjunction with active disease treatment.

Given the high risks and poor outcomes of palliation in advanced stage pancreatic cancer, appropriate risk stratification is essential. In addition, treatment decisions must be made in conjunction with the patient and their family, keeping their goals and priorities in mind. Although conceptually intuitive, ascertaining this information can be difficult. Models such as the "palliative care triangle", first published by Thomas Miner in 2002, can serve as a rubric for physicians to learn how to assess the patient's and family's concerns, values, and emotions when evaluating the risks and benefits with available medical and surgical treatment options. [27, 28] (Figure 2) Use of this model fosters strong relationships amongst all parties involved in treatment decisions, allowing for shared decision-making and clear expectations as various treatment options are chosen.

When used in conjunction with risk stratification, the palliative care model helps treating physicians understand a patient's'disease characteristics, prognosis, expectations, and goals of treatment in order to choose the best treatment when considering the various options available for the management of the most common 
sequelae of advanced pancreatic cancer: obstructive jaundice, gastric outlet obstruction, and pain.

\section{Obstructive Jaundice}

Jaundice is a presenting symptom in $51-72 \%$ of patients with unresectable pancreatic cancer and develops in over $80 \%$ of patients during the natural history of their disease. $[2,3,29,30]$ Obstructive jaundice results in pruritus and fat malabsorption due to decreased excretion of bile acids into the duodenum, causing anorexia and diarrhea, and worsening malnutrition and cachexia. Fat malabsorption also decreases the ability to absorb fat-soluble vitamins, including vitamin $\mathrm{K}$, leading to coagulopathy, and increased risk of bleeding. [31]

Unrelieved cholestasis can lead to hepatic dysfunction and even liver failure. Patients with biliary obstruction are at increased risk for cholangitis, particularly if the biliary tree was previously instrumented and not appropriately drained. In one series, up to $38 \%$ of patients without any palliative treatment died from complications of biliary obstruction.[3]

\section{Planned Operative and Endoscopic Palliation}

Historically operative biliary-enteric bypass was standard practice for locally advanced, unresectable disease. (Figure 4). Options including Roux-en-Y hepaticoor choledochojejunostomy, were most commonly performed. Surgical palliation with cholecystojejunostomy or choledochoduodenostomy fell out of favor due to a high probability of cystic duct, duodenal or gastric outlet obstruction by the tumor over time, as well as the possibility of bile reflux into the stomach.

In the 1990s, endoscopic interventions for the diagnosis and palliation of obstructive jaundice became more widely available. Currently, the placement of endoscopic biliary stents during endoscopic retrograde cholangiopancreatography (ERCP) is the preferred method for palliation of obstructive jaundice in patients with unresectable or metastatic pancreatic cancer. Advances in endoscopic technology have led to successful stent placement in more than $90 \%$ of patients during ERCP with equal efficacy, but less morbidity and mortality when compared 
to surgical palliation with biliary-enteric bypass. [3, 32, 33] Although less invasive than surgical palliation, endostent placement has associated risks including cholangitis (35\%), acute pancreatitis (29\%), bleeding (23\%), perforation (6\%), and early stent migration (3\%). [3, 33]

There are a number of different endostents available, each with its own benefits and limitations. Self-expanding metal stents (SEMS) have a longer patency, with covered metal stents lasting 7-10 months owing to their ability to withstand tumor ingrowth. $[3,31,33]$ Stent migration occurs up to $20 \%$ of the time and can occur with any stent type $[3,33]$. Compared to covered metal stents, uncovered metal stents migrate less frequently [34], but can theoretically be occluded by tumor ingrowth (as shown in Figure 3). Recent innovations in stent technology have led to the development of covered and uncovered SEMS with low axial force and flared ends. A 2013 randomized multicenter trial utilizing these newer stents showed no evidence of stent migration as well as longer periods without stent dysfunction in the covered stent cohort. [35]

Plastic stents are small and prone to obstruction secondary to biliary sludge and bacterial overgrowth, with a median patency of only 2-4 months. [3, 31, 33]Although plastic stents are significantly less expensive than metal stents, overall costs become lower with SEMS if a patient survives $>4$ months due to fewer repeat procedures and hospitalizations so selection should depend upon a patient's prognosis.[3] Therefore, in general it is recommended that patients with expected survival greater than 4 months have SEMS placed (Figure 5).

As survival improves with newer chemotherapeutic agents, the role of operative biliary-enteric bypass may change. While the previously discussed studies reported lower early complication rates with endoscopic stenting compared to operative bypass, stented patients have higher rates of later complications such as recurrent jaundice (36\% vs. $2 \%$ ) requiring reintervention[36]. In these patients who remain unresectable but have a good good performance status, operative intervention can be considered in the setting of repeated episodes of stentassociated complications, operative intervention can be considered. However, 
studies still show the majority of patients who are stented have an improved quality of life, even with the need for multiple stent changes and higher rates of recurrent.

\section{Unresectable disease found at laparotomy.}

Prior to the development of thin cut cross sectional imaging in the 1990s, nearly $30-40 \%$ of patients undergoing surgical exploration for resection were found to have locally advanced or metastatic disease.[2, 3] In these cases, surgical palliation included a hepaticojejunostomy and gastrojejunostomy ("double bypass") in combination with ethanol ablation of the celiac neural plexus (chemical splanchnicectomy). Although improvements in pre-operative imaging and staging have decreased the number of patients of unresectable patients identified at exploration, up to $10 \%$ of patients are still found to have locally advanced or metastatic disease at the time of operation.[2, 3]

The management of the obstructed biliary tree in the setting of unexpected metastatic or locally advanced disease is controversial. Options include proceeding with operative palliation or closing the abdomen and pursuing endoscopic interventions if the patient did not already undergo preoperative biliary drainage. Unfortunately, there are no definitive data describing survival and quality of life of patients undergoing surgical bypass versus closure and rapid placement of endoscopic stents. For this reason, when faced with this decision, the surgeon's understanding of a patient's symptoms, functional status, and projected survival are crucial. In the setting of carcinomatosis or multi-focal metastatic disease, regardless of performance status endoscopic intervention should be favored due to the short median survival. However, in those patients with good functional status and low volume metastatic disease or locally advanced disease, operative bypass is a reasonable option. Figure 5 provides an algorithm for the management of pancreatic malignancy.

Special Cases Requiring Percutaneous Transhepatic Palliation of Obstructive Jaundice

When endoscopic stent placement is unsuccessful or when anatomical constraints make endoscopic biliary cannulation difficult (i.e. previous gastric 
bypass surgery or recurrence following pancreaticoduodenectomy), a transhepatic biliary catheter or metal stent can be placed by accessing the bile ducts with a needle percutaneously through the liver parenchyma. A wire is then advanced past the obstruction allowing for the placement of biliary drains or metallic stents similar to those placed endoscopically. If a lesion cannot be traversed, a catheter(s) can be left proximal to the obstruction to drain the biliary tree. This approach is often required in the setting of recurrent tumor at the hepaticojejunostomy after pancreaticoduodenectomy.

\section{Gastric Outlet Obstruction}

Ten to twenty-five percent of patients with pancreatic cancer will develop signs and symptoms of gastric outlet obstruction (GOO) requiring intervention prior to their death $[3,31,42,43]$ and an estimated $20 \%$ of patients will die with symptoms of duodenal obstruction. [43] Patients experience nausea and vomiting, resulting in decreased oral intake, electrolyte disturbances, and subsequent malnutrition and cachexia. Due to the impact on quality of life, addressing this is paramount although obtaining symptom resolution is challenging once the disease has progressed to this extent.

\section{Surgical Palliation-Gastrojejunostomy}

Historically, the gold standard for management of gastric outlet obstruction (GOO) was the creation of a gastrojejunostomy. The procedure is performed by bringing up a loop of jejunum approximately $20-30 \mathrm{~cm}$ distal to the ligament of Treitz and performing a 3-4 cm side-to-side gastrojejunal anastomosis. This can be performed ante- or retrocolic, although the former is usually easier and also

prevents obstruction of the loop by metastatic adenopathy at the root of the mesentery. If possible, the anastomosis should be performed on the posterior aspect of the stomach in order to improve emptying except in the case of large bulky tumors, where the anastomosis should be made on the anterior surface of the stomach to prevent re-obstruction. The most common complication associated with 
creation of a gastrojejunostomy is delayed gastric emptying, which occurs in up to $30-50 \%$ of patients, leaving them with ongoing nausea, vomiting, and difficulty eating. $[44,45]$

There are limited data directly comparing laparoscopic to open gastrojejunostomy for malignant G0O. However, in a small cohort of 24 patients with malignant GOO randomized to laparoscopic or open gastrojejunostomy, Narvarra et al found the laparoscopic approach resulted in decreased time to oral intake (4.08 vs 6.25 days) and a trended towards decreased rates of delayed gastric emptying and length of stay.[46]

\section{Prophylactic Gastrojejunostomy}

Although it is generally accepted that a completely asymptomatic patient would not be explored to perform a prophylactic gastrojejunostomy, controversy exists on whether to perform a gastrojejunostomy in the absence of symptoms when metastatic or locally advanced disease is discovered during the time of exploration. Two prospective, randomized trials evaluated the role of prophylactic gastrojejunostomy in patients found to be unresectable at the time of surgery without symptoms of GOO preoperatively. Prophylactic gastrojejunostomy was associated with a decreased incidence of late GOO with no difference in postoperative complications and length of stay between the two groups. $[47,48]$ Similarly, a 2013 Cochrane review concluded that routine prophylactic gastrojejunostomy was indicated in patients with unresectable periampullary cancer already undergoing exploratory laparotomy (with or without biliary bypass).[49] However caution in the interpretation of these studies must be exercised as these studies have an inherent selection bias. The overall survival and outcomes for patients who have not yet developed GOO are superior to those patients with symptomatic locally advanced disease. Also, it is unclear how many of these asymptomatic patients actually would have gone on to develop symptoms requiring intervention. Therefore, the universal adoption of prophylactic gastrojejunostomy is not recommended, but instead should be determined on caseby-case basis (Figure 5). Similar to hepaticojejunostomy, the presence of 
carcinomatosis or metastatic disease should discourage surgeons from performing gastrojejunostomy in asymptomatic patients. As always treatment decisions need to weigh the operative risks, duration of survival, and effectiveness of the intervention.

\section{Endoscopic Palliation}

With advances in endoscopic techniques, management of gastric outlet obstruction can be performed endoscopically, with placement of large selfexpanding stents, typically $20-22 \mathrm{~mm}$ in diameter, and $60-90 \mathrm{~mm}$ in length. Stents are successful in $92-100 \%$ of cases allowing patients to resume oral intake in as little as 24 hours in $73-93 \%$ of patients. [3, 33] Although patients can manage their own salivary secretions and drink liquids, stent occlusion often occurs with solid food. Early complications occur in $2-12 \%$ of cases $[33,42]$ and include perforation, GI hemorrhage, aspiration pneumonia, or jaundice and potential cholangitis from compression of the common bile duct. [33] Overall complications occur in $26 \%$ of patients including late complications, such as stent failure and migration. [3] Stent

placement should be reserved for symptomatic patients, as there is a high risk of migration if the stent is placed in non-critical stenosis.[33]

\section{Endoscopic Stenting Versus Gastrojejunostomy}

The decision to proceed with stenting or surgery for malignant G00 depends in large part on the patient's predicted survival time and functional status. Early studies reported similar success rates between stenting and surgery. Stented patients had fewer complications, faster return to oral intake, and shorter hospitalizations. [50-53] However, the surgical gastrojejunostomy or endoscopic stent placement for the palliation of malignant gastric outlet obstruction (SUSTENT) trial, a multicenter randomized control trial comparing stenting to surgery, found that although endoscopic palliation showed a quicker return to oral intake (5 vs. 8 days), at 30 and 60 days the surgical group had significantly better oral intake. After adjusting for survival, surgery was associated with more total days tolerating oral intake and is recommended for patients with a life expectancy of 2 months or longer 
(Figure 5). [54] However, many surgeons would advocate for reserving operative intervention for those patients with a life expectancy of at least 4 months and a good functional status.

In our experience, even when a technically sound anastomosis is performed, or a patent stent is in place, a stomach that has been chronically obstructed, may not fully recover function. As there are no data predicting which patient populations will benefit, expectations of any intervention need to be tempered. Additionally, when a patient with poor functional status presents with a malignant gastric outlet obstruction from pancreatic cancer, outcomes with either procedure are routinely poor with a median survival of 2 months.[55] In this setting, another option that should be considered is placement of a decompressive PEG to allow for pleasure feeds, or direct consultation of hospice services for symptom control.

\section{Pain Management}

Seventy-five percent of patients will present with abdominal and/or back pain and $80 \%$ of patients with advanced pancreatic cancer will experience severe pain prior to death commonly associated with malignant invasion of the celiac plexus. $[1,31,56]$ While many patients are treated with oral and transdermal analgesics, there is evidence that patients who undergo early regional neurolysis of the celiac plexus experience improved quality of life. $[3,56]$ A 2011 Cochrane metaanalysis of 6 randomized controlled trials, found that celiac plexus block (chemical splanchnicectomy) results in a small but significant decrease in pain at 4 and 8 weeks post procedure. Additionally they found a durable decrease in overall opioid consumption and associated side effects. [57]

There are four ways to perform a celiac block: intraoperatively, percutaneously, endoscopically or thoracoscopically. Intraoperative celiac block is performed by injecting ethanol or a local anesthetic to either side of the aorta at the level of celiac axis. In a double blind randomized control trial by Lillemoe et al, [58] intraoperative celiac block (chemical splanchnicectomy) produced a statistically significant decrease in pain and opioid consumption, when compared to saline control. In a meta-analysis from 2009, patients undergoing endoscopic ultrasound 
guided celiac plexus blocks had an $85 \%$ response rate to therapy (measured by improvement in pain score) and a low complication rate. [59] Similar response rates have been seen in small series evaluating thoracoscopic celiac blocks.[3]

Celiac plexus blocks can result in urinary retention, back pain, and diarrhea occurring in about $38 \%$ of patients, resulting from unopposed parasympathetic tone. [60,61] Patients can also experience transient orthostatic hypotension secondary to vasodilation (1-3\%) which can last almost a week. [60] More serious, but rare complications include transient or permanent paraplegia, abdominal aortic dissection or retroperitoneal hemorrhage. $[60,61]$

\section{Other Issues in Pancreatic Cancer}

Nutrition and Pancreatic Exocrine insufficiency

Pancreatic cancer patients experience cachexia due to appetite loss, malnutrition, and hypercatabolism of lean tissue, leading to weakness, fatigue, and a poor quality of life. One modifiable factor is pancreatic exocrine insufficiency, resulting from pancreatic duct obstruction or replacement of the pancreatic parenchyma by malignant cells, gland fibrosis or atrophy. [31] Sixty-five percent of

patients with pancreatic cancer will experience symptoms of fat malabsorption, and $50 \%$ will also have protein malabsorption.[1,61] Treatment of patients with pancreatic enzyme replacement has been found to improve malabsorption, bloating, and diarrhea, as well as prevent further weight loss. [1, 31, 62] In a randomized controlled trial, patients with unresectable pancreatic cancer who took enzyme replacements for 8 weeks were found to have a 1.2\% increase in body weight, compared to placebo patients who had a 3.7\% decrease in body weight.[62]

\section{Thromboembolic Disease}

A 2004 analysis of the Medicare claims database showed that pancreatic cancer patients have one of the highest incidences of deep venous thrombosis (DVT) or pulmonary embolism (PE) with rates ranging from $17-57 \%$ with a relative risk of VTE of 8.8 (95\% CI 3.5-22.4; $\mathrm{p}=-0.001)$. This high rate was seen even when 
excluding patients with vascular access thrombosis or direct tumor related vascular invasion. [63]

Although the presence of thromboembolic disease is associated with a worse prognosis, treatment can improve longevity and symptomatology. Patients with acute portal vein thrombosis (symptoms less than 14 days prior to presentation) can be selectively treated with portal thrombolytic therapy as propagation to the superior mesenteric vein can be deadly. Otherwise, treatment for all acute venous thromboembolic disease includes treatment with LWMH, unfractionated heparin, or oral anticoagulation. The use of oral anticoagulants such as warfarin must be carefully considered as factors such as malnutrition, liver dysfunction, chemotherapy, and antibiotics can all disrupt the vitamin K metabolism making it difficult to achieve steady levels in these patients. The 2003 CLOT study (Randomized Comparison of Low-Molecular-Weight Heparin versus Oral Anticoagulant Therapy for the Prevention of Recurrent Venous Thromboembolism in Patients with Cancer Study) compared the use of LMWH to oral anticoagulation (dalteparin vs a warfarin derivative) in patients with acute DVT and malignancy. They found no difference in bleeding complications between the two groups, but patients on the warfarin derivative had an increased risk of recurrent thromboembolic disease compared to those on dalteparin $(17 \%$ vs. $9 \%, \mathrm{HR}=0.48$, $\mathrm{p}=0.002$ ). Additionally, patients on the warfarin derivative had an INR in the therapeutic range for only $46 \%$ of the 6-month study time. [64] Newer generation oral anticoagulants are currently being studied for their use in this patient population. Once diagnosed with VTE, many patients will remain on anticoagulation until reaching the end of life.

\section{End of Life Care and the Role of Hospice}

The aggressive nature and particularly poor prognosis associated with pancreatic adenocarcinoma can leave patients and families feeling blind-sided when suddenly faced with end of life decisions. Therefore, it is crucial to clarify the patient's and family's goals from the onset and address misconceptions they have about the various treatment options and survival. These conversations can be the 
most time consuming and difficult portions of patient care, but establishing clear goals early on avoids unnecessary complications, cost and conflict, especially as goals and attitudes evolve with changing prognosis. When discussing potential interventions it is critical to discuss not only prognosis, but also the potential benefits and harms of every option. Unfortunately, little information is available on outcomes such as quality of life, time spent in the hospital, and other outcomes that may be important to patients, thereby limiting our ability to help patients make informed decisions.

Analysis of the Surveillance, Epidemiology, and End Results (SEER)-Medicare linked data of 25,476 patients with pancreatic cancer from 1992-2005, found that across all treatment groups, hospital days increased at the end of life, suggesting the significant use of resources when they are least likely to benefit patients. Furthermore, in a population-based study of end-of-life care in pancreatic cancer patients, Sheffield et al. found an increase in aggressive care in the last month of life, with an increase in ICU admission from 15.5\% in 1992-1994 to $19.6 \%$ in 2004-2006 $(\mathrm{P}<0.0001)$ and an increase in receipt of chemotherapy from $8.1 \%$ to $16.4 \%$ $(\mathrm{P}<0.0001) .[65]$ This suggests that aggressive care, in addition to being linked to worse quality of life for patients, and worse bereavement adjustment for their caregivers, [66-69] results in increased costs with little survival benefit.

Despite the fact that enrollment in hospice care has been associated with lower Medicare costs, and improved quality of life, including improved pain, less fatigue, and lower depression and anxiety rates, it is grossly underutilized. [70-72] In the same population-based study of end-of -life care in pancreatic cancer patients only $56 \%$ of patients were enrolled in hospice prior to death, with only $35.9 \%$ of hospice users enrolled for four weeks or more, suggesting that more than half of patients with pancreatic cancer are not receiving the full benefit of hospice care. Furthermore, among patients with loco-regional disease, those who underwent resection were less likely to enroll in hospice before death, supporting the need for early discussion of palliative care and hospice care regardless of stage at diagnosis. [65] 
The early introduction of hospice care can help normalize the concept to patients and families, and give them ample time to clarify any misconceptions. Moreover, it is possible that a paradigm shift may occur where the receipt of hospice care may no longer mean having to forgo potentially curative therapies. An ongoing pilot study amongst Medicare patients with advanced cancer allows patients to receive potentially curative treatment in conjunction with hospice care and supportive services. [73] Results of pilot studies such as these may positively impact care on many levels, leading to more time spent with family at the end of life instead of in the hospital.

\section{Conclusion}

The involvement of patients, families, physicians, and advocacy groups throughout the care process beginning at the time of diagnosis and utilizing the palliative care triangle to clarify a patient's and family's goals allows for the interpretation of potential outcomes in ways that are meaningful to all stakeholders. This allows patients with pancreatic cancer and their physicians to choose "the right treatment, for the right patient, in the right setting." More importantly these discussions provide an opportunity to reframe how patients and families think of hope, which is central to all who are dealing with cancer. Instead of creating a situation where families feel robbed of hope when we can no longer offer cure, early discussions can focus on ways to empower patients to create new achievable goals. For some, this will be to die in the comfort of their home or to feel well enough to attend an important family event, instead of continuing treatment that will ultimately not change their overall outcome. When given new goals to focus on, hope is reborn and patients, families, and providers benefit.

\section{REFERENCES}


1. el-Kamar, F.G., M.L. Grossbard, and P.S. Kozuch, Metastatic pancreatic cancer: emerging strategies in chemotherapy and palliative care. Oncologist, 2003. 8(1): p. 18-34.

2. Kneuertz, P.J., et al., Palliative surgical management of patients with unresectable pancreatic adenocarcinoma: trends and lessons learned from a large, single institution experience. J Gastrointest Surg, 2011. 15(11): p. 191727.

3. Stark, A. and O.J. Hines, Endoscopic and operative palliation strategies for pancreatic ductal adenocarcinoma. Semin Oncol, 2015. 42(1): p. 163-76.

4. Loehrer, P.J., Sr., et al., Gemcitabine alone versus gemcitabine plus radiotherapy in patients with locally advanced pancreatic cancer: an Eastern Cooperative Oncology Group trial. J Clin Oncol, 2011. 29:4105e12.

5. Gourgou-Bourgade, S., et al., Impact of FOLFIRINOX compared with gemcitabine on quality of life in patients with metastatic pancreatic cancer: results from the PRODIGE 4/ACCORD 11 randomized trial. J Clin Oncol, 2013. 31(1): p. 23-9.

6. Singhal, M., et al., A phase III trial comparing FOLFIRINOX versus gemcitabine for metastatic pancreatic cancer. Ann Oncol, 2014. 25(suppl 4): p. iv210iv211.

7. Conroy, T., et al., FOLFIRINOX versus gemcitabine for metastatic pancreatic cancer. N Engl J Med, 2011. 364(19): p. 1817-25.

8. Moertel, C.G., et al., Therapy of locally unresectable pancreatic carcinoma: a randomized comparison of high dose (6000 rads) radiation alone, moderate dose radiation (4000 rads +5 -fluorouracil), and high dose radiation +5 fluorouracil: The Gastrointestinal Tumor Study Group. Cancer, 1981. 48(8): p. 1705-10.

9. Shinchi, H., et al., Length and quality of survival after external-beam radiotherapy with concurrent continuous 5-fluorouracil infusion for locally unresectable pancreatic cancer. Int J Radiat Oncol Biol Phys, 2002. 53(1): p. 146-50.

10. Sultana, A., et al., Systematic review, including meta-analyses, on the management of locally advanced pancreatic cancer using radiation/combined modality therapy. Br J Cancer, 2007. 96(8): p. 1183-90.

11. Hammel, P., et al., Comparison of chemoradiotherapy (CRT) and chemotherapy (CT) in patients with a locally advanced pancreatic cancer (LAPC) controlled after 4 months of gemcitabine with or without erlotinib: final results of the inter- national phase III LAP 07 study. J Clin Oncol, 2013. 31(Suppl): p. abstr LBA4003.

12. Winter, J.M., et al., Survival after resection of pancreatic adenocarcinoma: results from a single institution over three decades. Ann Surg Oncol, 2012. 19(1): p. 169-75.

13. Konstantinidis, I.T., et al., Pancreatic ductal adenocarcinoma: is there a survival difference for $R 1$ resections versus locally advanced unresectable tumors? What is a "true" R0 resection? Ann Surg, 2013. 257(4): p. 731-6.

14. Stathis, A. and M.J. Moore, Advanced pancreatic carcinoma: current treatment and future challenges. Nat Rev Clin Oncol, 2010. 7(3): p. 163-72. 
15. Dimou, F., et al., Trends in Receipt and Timing of Multimodality Therapy in Early-Stage Pancreatic Cancer. J Gastrointest Surg, 2016. 20(1): p. 93-103.

16. Evans, D.B., et al., Preoperative gemcitabine-based chemoradiation for patients with resectable adenocarcinoma of the pancreatic head. J Clin Oncol, 2008. 26(21): p. 3496-502.

17. Varadhachary, G.R., et al., Preoperative gemcitabine and cisplatin followed by gemcitabine-based chemoradiation for resectable adenocarcinoma of the pancreatic head. J Clin Oncol, 2008. 26(21): p. 3487-95.

18. Christians, K.K., et al., Survival of patients with resectable pancreatic cancer who received neoadjuvant therapy. Surgery, 2016. 159(3): p. 893-900.

19. Balcom, J.H.t., et al., Ten-year experience with 733 pancreatic resections: changing indications, older patients, and decreasing length of hospitalization. Arch Surg, 2001. 136(4): p. 391-8.

20. Winter, J.M., et al., 1423 pancreaticoduodenectomies for pancreatic cancer: $A$ single-institution experience. J Gastrointest Surg, 2006. 10(9): p. 1199-210; discussion 1210-1.

21. Reddy, D.M., et al., Readmission after pancreatectomy for pancreatic cancer in medicare patients. J Gastrointest Surg, 2009. 13(11): p. 1963-74; discussion 1974-5.

22. Riall, T.S., et al., The effect of age on short-term outcomes after pancreatic resection: a population-based study. Ann Surg, 2008. 248(3): p. 459-67.

23. Le Pechoux, C., et al., Standard-dose versus higher-dose prophylactic cranial irradiation (PCI) in patients with limited-stage small-cell lung cancer in complete remission after chemotherapy and thoracic radiotherapy (PCI 99-01, EORTC 22003-08004, RTOG 0212, and IFCT 99-01): a randomised clinical trial. Lancet Oncol, 2009. 10(5): p. 467-74.

24. Gillen, S., et al., Preoperative/neoadjuvant therapy in pancreatic cancer: a systematic review and meta-analysis of response and resection percentages. PLoS Med, 2010. 7(4): p. e1000267.

25. World Health Organization . World Health Organization . Available at: http://www.who.int/cancer/palliative/definition/en/. Accessed December 12.

26. Temel, J.S., et al., Early palliative care for patients with metastatic non-smallcell lung cancer. N Engl J Med, 2010. 363(8): p. 733-42.

27. Miner, T.J., et al., The palliative triangle: improved patient selection and outcomes associated with palliative operations. Arch Surg, 2011. 146(5): p. $517-22$.

28. Miner, T.J., Communication skills in palliative surgery: skill and effort are key. Surg Clin North Am, 2011. 91(2): p. 355-66, ix.

29. Lyons, J.M., et al., Operative procedures for unresectable pancreatic cancer: does operative bypass decrease requirements for postoperative procedures and in-hospital days? HPB (Oxford), 2012. 14(7): p. 469-75.

30. Crippa, S., et al., Quality of life in pancreatic cancer: analysis by stage and treatment. J Gastrointest Surg, 2008. 12(5): p. 783-93; discussion 793-4.

31. Nakakura, E.K. and R.S. Warren, Palliative care for patients with advanced pancreatic and biliary cancers. Surg Oncol, 2007. 16(4): p. 293-7. 
32. House, M.G. and M.A. Choti, Palliative therapy for pancreatic/biliary cancer. Surg Clin North Am, 2005. 85(2): p. 359-71.

33. Maire, F. and A. Sauvanet, Palliation of biliary and duodenal obstruction in patients with unresectable pancreatic cancer: endoscopy or surgery? J Visc Surg, 2013. 150(3 Suppl): p. S27-31.

34. Almadi, M.A., A.N. Barkun, and M. Martel, No benefit of covered vs uncovered self-expandable metal stents in patients with malignant distal biliary obstruction: a meta-analysis. Clin Gastroenterol Hepatol, 2013. 11(1): p. 2737 e1.

35. Kitano, M., et al., Covered self-expandable metal stents with an anti-migration system improve patency duration without increased complications compared with uncovered stents for distal biliary obstruction caused by pancreatic carcinoma: a randomized multicenter trial. Am J Gastroenterol, 2013. 108(11): p. 1713-22.

36. Smith, A.C., et al., Randomised trial of endoscopic stenting versus surgical bypass in malignant low bileduct obstruction. Lancet, 1994. 344(8938): $\mathrm{p}$. 1655-60.

37. Moss, A.C., E. Morris, and P. Mac Mathuna, Palliative biliary stents for obstructing pancreatic carcinoma. Cochrane Database Syst Rev, 2006(2): p. CD004200.

38. Artifon, E.L., et al., Surgery or endoscopy for palliation of biliary obstruction due to metastatic pancreatic cancer. Am J Gastroenterol, 2006. 101(9): p. 2031-7.

39. Maire, F., et al., Long-term outcome of biliary and duodenal stents in palliative treatment of patients with unresectable adenocarcinoma of the head of pancreas. Am J Gastroenterol, 2006. 101(4): p. 735-42.

40. Mortenson, M.M., H.S. Ho, and R.J. Bold, An analysis of cost and clinical outcome in palliation for advanced pancreatic cancer. Am J Surg, 2005. 190(3): p. 406-11.

41. Sarr, M.G. and J.L. Cameron, Surgical management of unresectable carcinoma of the pancreas. Surgery, 1982.91(2): p. 123-33.

42. Schmidt, C., et al., A prospective observational study examining quality of life in patients with malignant gastric outlet obstruction. Am J Surg, 2009. 198(1): p. 92-9.

43. Sohn, T.A., et al., Surgical palliation of unresectable periampullary adenocarcinoma in the 1990s. J Am Coll Surg, 1999. 188(6): p. 658-66; discussion 666-9.

44. Oida, T., et al., A novel technique of laparoscopic gastrojejunostomy-modified Devine exclusion with vertical stomach reconstruction-for gastric outlet obstruction to preventing blow-out of the distal gastric remnant and delayed in return of gastric emptying. Hepatogastroenterology, 2009. 56(89): p. 282-4.

45. Usuba, T., et al., Is modified Devine exclusion necessary for gastrojejunostomy in patients with unresectable pancreatobiliary cancer? Surg Today, 2011. 41(1): p. 97-100. 
46. Navarra, G., et al., Palliative antecolic isoperistaltic gastrojejunostomy: a randomized controlled trial comparing open and laparoscopic approaches. Surg Endosc, 2006. 20(12): p. 1831-4.

47. Lillemoe, K.D., et al., Is prophylactic gastrojejunostomy indicated for unresectable periampullary cancer? A prospective randomized trial. Ann Surg, 1999. 230(3): p. 322-8; discussion 328-30.

48. Van Heek, N.T., et al., The need for a prophylactic gastrojejunostomy for unresectable periampullary cancer: a prospective randomized multicenter trial with special focus on assessment of quality of life. Ann Surg, 2003. 238(6): p. 894-902; discussion 902-5.

49. Gurusamy, K.S., S. Kumar, and B.R. Davidson, Prophylactic gastrojejunostomy for unresectable periampullary carcinoma. Cochrane Database Syst Rev, 2013. 2: p. CD008533.

50. Fiori, E., et al., Palliative management of malignant antro-pyloric strictures. Gastroenterostomy vs. endoscopic stenting. A randomized prospective trial. Anticancer Res, 2004. 24(1): p. 269-71.

51. Gaidos, J.K. and P.V. Draganov, Treatment of malignant gastric outlet obstruction with endoscopically placed self-expandable metal stents. World J Gastroenterol, 2009. 15(35): p. 4365-71.

52. Chandrasegaram, M.D., et al., Endoscopic stenting versus operative gastrojejunostomy for malignant gastric outlet obstruction. Surg Endosc, 2012. 26(2): p. 323-9.

53. Rudolph, H.U., et al., Malignant gastroduodenal obstruction: retrospective comparison of endoscopic and surgical palliative therapy. Scand J Gastroenterol, 2011. 46(5): p. 583-90.

54. Jeurnink, S.M., et al., Surgical gastrojejunostomy or endoscopic stent placement for the palliation of malignant gastric outlet obstruction (SUSTENT study): a multicenter randomized trial. Gastrointest Endosc, 2010. 71(3): p. 490-9.

55. Oh, S.Y., et al., Survival and clinical outcome after endoscopic duodenal stent placement for malignant gastric outlet obstruction: comparison of pancreatic cancer and nonpancreatic cancer. Gastrointest Endosc, 2015. 82(3): p. 460-8 e2.

56. Wong, G.Y., et al., Effect of neurolytic celiac plexus block on pain relief, quality of life, and survival in patients with unresectable pancreatic cancer: $a$ randomized controlled trial. JAMA, 2004. 291(9): p. 1092-9.

57. Arcidiacono, P.G., et al., Celiac plexus block for pancreatic cancer pain in adults. Cochrane Database Syst Rev, 2011(3): p. CD007519.

58. Lillemoe, K.D., et al., Chemical splanchnicectomy in patients with unresectable pancreatic cancer. A prospective randomized trial. Ann Surg, 1993. 217(5): p. 447-55; discussion 456-7.

59. Puli, S.R., et al., EUS-guided celiac plexus neurolysis for pain due to chronic pancreatitis or pancreatic cancer pain: a meta-analysis and systematic review. Dig Dis Sci, 2009. 54(11): p. 2330-7.

60. Erdek, M.A., L.M. King, and S.G. Ellsworth, Pain management and palliative care in pancreatic cancer. Curr Probl Cancer, 2013. 37(5): p. 266-72. 
61. Fazalova, V.P., et al., [Effect of demographic population factors and individual biological parameters on the rate of neutral molecular evolution]. Genetika, 2007. 43(9): p. 1172-80.

62. Bruno, M.J., et al., Placebo controlled trial of enteric coated pancreatin microsphere treatment in patients with unresectable cancer of the pancreatic head region. Gut, 1998. 42(1): p. 92-6.

63. Khorana, A.A. and R.L. Fine, Pancreatic cancer and thromboembolic disease. Lancet Oncol, 2004. 5(11): p. 655-63.

64. Lee, A.Y., et al., Low-molecular-weight heparin versus a coumarin for the prevention of recurrent venous thromboembolism in patients with cancer. $\mathrm{N}$ Engl J Med, 2003. 349(2): p. 146-53.

65. Sheffield, K.M., et al., End-of-life care in Medicare beneficiaries dying with pancreatic cancer. Cancer, 2011. 117(21): p. 5003-12.

66. Wright, A.A., et al., Associations between end-of-life discussions, patient mental health, medical care near death, and caregiver bereavement adjustment. JAMA, 2008. 300(14): p. 1665-73.

67. Wright, A.A., et al., Place of death: correlations with quality of life of patients with cancer and predictors of bereaved caregivers' mental health. J Clin Oncol, 2010. 28(29): p. 4457-64.

68. Earle, C.C., et al., Identifying potential indicators of the quality of end-of-life cancer care from administrative data. J Clin Oncol, 2003. 21(6): p. 1133-8.

69. Grunfeld, E., et al., Toward population-based indicators of quality end-of-life care: testing stakeholder agreement. Cancer, 2008. 112(10): p. 2301-8.

70. Bischoff, K., V. Weinberg, and M.W. Rabow, Palliative and oncologic comanagement: symptom management for outpatients with cancer. Support Care Cancer, 2013. 21(11): p. 3031-7.

71. Mitchell, S.L., et al., Hospice care for patients with dementia. J Pain Symptom Manage, 2007. 34(1): p. 7-16.

72. Greer, D.S., et al., An alternative in terminal care: results of the National Hospice Study. J Chronic Dis, 1986. 39(1): p. 9-26.

73. Centers for Medicare \& Medicaid Services. Centers for Medicare \& Medicaid Services. Available at: https://innovation.cms.gov/initiatives/medicare-carechoices/. Accessed December 20. 


\section{Figure Legends}

Figure 1: Enhanced model of Palliative Care. Palliative care and hospice are crucial components along the spectrum of disease and ultimately death. From the time of diagnosis to the point of death, the focus and modality of care change from curative treatments to comfort care and hospice. Palliation is a crucial bridge between these two modalities, providing not only palliation for pain and symptoms but also continuing to seek curative therapies.

Figure 2: Palliative Triangle. The palliative triangle emphasizes the equal involvement between the physician, the patient, and their family. Each participant brings his/her own goals and desires to the conversation, and it is these individual desires and factors that help guide the treatment decisions. Ultimately, the triangle aims to unify all the participants in their desire for the good of the patient.

Figure 3: A. Bare Metal Stent placement and accompanying ERCP with stent not maximally expanded. B. Tumor ingrowth through the stent including necrotic debris. ERCP shows fully expanded metal stent with proximal dilated biliary tree due to stent occlusion. C. Covered metal stent placed with completion ERCP showing patent stent and well draining biliary tree.

Figure 4: Operative palliation for biliary obstruction involves the formation of a roux-en-y hepaticojejunostomy or choledochojejunostomy (not shown) with or without formation of a gastrojejeunostomy to palliate gastric outlet obstruction (also not shown).

Figure 5: Algorithm for the management of biliary and/or gastric outlet obstruction in pancreatic adenocarcinoma. 